Article

\title{
Depression Risks and Correlates among Different Generations of Chinese Americans: The Effects of Relationships with Friends and Relatives
}

\author{
Lin Zhu \\ lin.zhu@temple.edu; Tel.: +1-267-324-9754 \\ Academic Editor: Brea L. Perry \\ Received: 10 March 2017; Accepted: 26 May 2017; Published: 3 June 2017
}

Center for Asian Health, Lewis Katz School of Medicine, Temple University, Philadelphia, PA 19122, USA;

\begin{abstract}
An increasing body of literature has suggested that the public portrayal of Chinese Americans as a high-achieving, well-adjusting "model minority" might not reflect the entire reality of their mental health conditions. This study examined depression risks and correlates among different generations of Chinese Americans, using non-Hispanic whites as a comparison group. A nationally representative sample of Chinese Americans $(n=600)$ from the Comprehensive Psychiatric Epidemiological Survey was used. Results of the study indicate that Chinese Americans in general have a lower risk of depression than non-Hispanic whites. Moreover, the prevalence and correlates of depression do not show a linear trend of difference from first to second to third-or-higher generation Chinese Americans, and then to non-Hispanic whites; rather, the risk of depression and its association with social relational factors presents in distinctive patterns for first and second generation Chinese Americans, compared to third-or-higher generation Chinese Americans and non-Hispanic whites. Specifically, friend network and relative group play different roles in influencing depression for different generations of Chinese Americans. The findings contributed to the growing body of literature on acculturation and mental health among immigrants, shedding lights on the complicated sociocultural contexts that could influence the mental well-being of individuals. Mental health service providers need to be aware of the complex and nuanced association between social relational factors and depression in their prevention, management, and treatment efforts.
\end{abstract}

Keywords: depression; social network; Chinese Americans; social support; social conflict; generational status

\section{Introduction}

Many empirical studies focus on immigrant minority groups because the process of migrating to a new country and adjusting to a new environment has complicated effects on the level and expression of distress, and access to and compliance with treatment (Tabora and Flaskerud 1994). Knowledge of sociocultural factors and their influence on the expression of distress and help-seeking behaviors is of primary importance in the assessments, diagnoses, and interventions made by both public and private health mental health professionals. The present study examines the prevalence and predictors of depression among Chinese Americans, using a nationally representative sample. Specifically, this study examines how chronic physical conditions, social support, and social conflict are associated with depression. The goal of this study is to better understand how important variables affect depression among different generations of Chinese Americans. Advanced knowledge of the issue can help identify high-risk subgroups within the community of Chinese Americans, and provide important insights for depression assessment, diagnosis, and treatment. 
The comparison between Chinese Americans and non-Hispanic whites is meaningful demographically, epidemiologically, and sociologically. The racial/ethnic composition of the population is constantly changing, especially in the US, which is why comparative research is necessary to advance our knowledge of the distribution of health needs among the current population. With non-Hispanic whites comprising the majority (63.7\%) of the US population (Hoeffel et al. 2012), how ethnic minority immigrants compare to non-Hispanic whites helps us estimate the health condition and need of the immigrant populations. Although Chinese Americans are usually touted by the media and the public as a high-achieving, well-adjusting "model minority" (Fong 2007), increasing evidence suggests that such public portrayals might not reflect the entire reality of their mental health conditions (Qin et al. 2008; Uba 2003). While some researchers have found lower rates of depression among Chinese Americans than among non-Hispanic whites (Takeuchi et al. 1998), others report no difference (Chen et al. 1998; Huang et al. 2006) or even the opposite (Ying 1988; Young et al.). The inconsistencies in literature could be attributed to the existence of methodological issues such as sample representativeness (mostly college students or local community samples), and variations in measures of depression. Moreover, the growing body of depression literature suggests depression risks among Asian Americans vary by immigration-related factors such as generational status, and sociocultural factors such as social support and conflicts (Kalibatseva and Leong 2011; Shen and Takeuchi 2001). In particular, social support and conflict has received increasing scholarly attention in depression literature (Stice et al. 2004; Wei et al. 2008). Their findings, though limited on conceptual and methodological fronts, suggest complicated interactions among various sociocultural factors in their impacts on depression.

\subsection{Generational Differences in Depression among Chinese Americans}

Immigration literature seems to have reached the consensus that acculturation is not a linear, one-directional model where the loss of the original culture occurs through greater acculturation to the host culture. Scholars point out that acculturation is a dynamic process of negotiating between maintaining original cultural characteristics and being involved in the larger society (Berry 1990; Berry 2003). However, the effects of the acculturation process on health outcomes at the individual-level are difficult to measure given the multifaceted and non-linear nature of the acculturation process. These measurement difficulties have similarly limited our understanding of the impact of culture on social networks, illness careers, and help-seeking behaviors within the larger social context (Nguyen and Bornheimer 2014). One approach that medical sociologists and social epidemiologists have taken is to examine the effects of proxy measures of acculturation, such as English proficiency, length of residency in the US, and generational status. Among these factors, generational status is one of the most studied. Immigrant generational status is defined by the nativity status of respondents and that of their parents. It has a strong empirical association with key direct measures of acculturation, including ethnic identification and language assimilation (Waters and Jiménez 2005). Immigrants who were born in a foreign country to foreign-born (first generation immigrants) are, in general, likely to have different immigrant experiences and socioeconomic trajectories compared to those who were born in the US to foreign-born parents (second generation), and to US-born individuals with US-born parents (third-or-higher generation) (Salant and Lauderdale 2003). In this sense, generational status is a concept that encompasses multiple factors related to acculturation, which means that knowledge of the generational differences in depression prevalence shed some light on the combined effect of the nativity status of an individual, nativity status of parents, English proficiency, and other sociocultural factors (Lee et al. 2016). Furthermore, for each specific ethnic group, differences across recent to early immigration generations present a unique pattern and reflects the complexity of immigration history and group socioeconomic status, among other things. Therefore, generational comparisons are more meaningful within one detailed ethnic group (e.g., Chinese Americans) than within an aggregated group (e.g., Asian Americans). 
According to the 2010 Census, over 76\% of Chinese Americans were foreign-born (Hoeffel et al. 2012), which means Chinese cultural values and beliefs are very likely to be prominent among the majority of Chinese Americans. Foreign-born Asian immigrants, especially those who arrived as adults, were less likely than the US-born Asian Americans to have depression or depressive symptoms. Comparisons between second and third-or-higher generation immigrants have been less often studied, and their findings are less than consistent (Takeuchi et al. 2007; Willgerodt and Thompson 2006). Nonetheless, scholars often attributed the generational disparities of depression to differences in demographic and socioeconomic characteristics. In addition, researchers noted the importance of examining how social relational factors such as coping resources vary across generations, and how such variation might help explain the generational difference in depression prevalence (Salant and Lauderdale 2003). Given these and other limitations discussed below, research has not yet been able to fully answer two key questions: (1) How does depression vary across different generations of immigrants? (2) Do sociodemographic and relational factors predict depression in the same way for different generations?

\subsection{Predictors of Depression among Chinese Americans}

Depression prevalence. There is a growing body of literature on depression prevalence and outcomes among individuals of Chinese cultural heritage, in mainland China, Hong Kong, as well Chinese immigrants in Canada and the United States. Yet, the observed depression rates were rather inconsistent. For example, the WHO Mental Health Survey data show that the lifetime prevalence of DSM-IV major depressive disorder is 3.5\% in two major Chinese cities, Beijing and Shanghai (Lee et al. 2007), which is much lower than the $17.1 \%$ prevalence in the US (Kessler et al. 1994). Other studies also report lower rates of depression in China, compared with the US (Kleinman 1982; Leung et al. 1992). Some scholars, however, found comparable or even higher level of CES-D depressive symptoms in Chinese samples when compared to the American samples (Cheung and Bagley 1998; Lin 1989). Inconsistency also exists in the literature on depression rates among Chinese Americans in the US. Some epidemiological studies reported a lower level of depression among Chinese Americans than non-Hispanic whites or predominantly white samples. For example, Takeuchi and colleagues (Takeuchi et al. 1998) reported a $6.9 \%$ lifetime prevalence of DSM-III-R major depressive disorder among 1747 Chinese Americans in Los Angeles, which is much lower than a national estimate of $17.1 \%$ in the National Comorbidity Survey using the same diagnostic measure (Kessler et al. 1994). Other studies also report a lower level of depression among Chinese Americans than that among non-Hispanic whites (Lai 2004; Kuo 1984). In contrast, Ying (1988) found that almost a quarter of her Chinese American sample were depressed, a proportion significantly higher than that of a predominantly white sample (7.94-9.25) in Radloff's study Radloff (1977). Such inconsistency in empirical findings is likely due to several critical differences in methodology and study design (measurement of depression, sampling strategies, or sample characteristics). The present study uses a nationally representative sample of Chinese American adults (18 or older), so that significant findings could be generalized to the greater Chinese American population in the US.

Sociodemographic factors. Previous studies of Chinese population found several significant predictors of depression. Specifically, elevated depression risk was found among women (Lee et al. 2009), older adults (Takeuchi et al. 1998; Hwang et al. 2000), those with lower income or more financial strains (Chou et al. 2004), and the unemployed (Takeuchi et al. 1998). The findings on the association between education and depression were less consistent. While Lai (Lai 2004) found a higher prevalence of depression among those with lower educational attainment among elderly Chinese Canadians, Mui (1999) found an association of the opposite direction among elderly Chinese Americans. Previous research has found significant differences in these sociodemographic factors (Portes and Rumbaut 2001; Schmid 2001), which is why it is necessary to control for them in this study.

Chronic physical conditions. In addition, previous studies of Chinese immigrants consistently found physical health status to be a strong predictor of depression (Lai 2004; Lee et al. 2009). 
One particularly noteworthy finding from a longitudinal study of Chinese Americans in Los Angeles showed that poor physical health status "both predated and occurred concomitantly with depressive episode" (Hwang et al. 2000). This finding was in line with previous hypotheses regarding the cultural variation in the depression symptomatology and somatic idioms in Chinese Americans (Uba 2003; Kleinman 1982). Specifically, the holistic orientation in traditional Chinese health philosophy may have resulted in a particularly strong association between physical health status and depression. Since a higher level of Chinese cultural subscription is more likely to be found among recent immigrants, the author hypothesizes that the association between chronic physical condition and depression should be stronger among first generation Chinese Americans than higher generation Chinese Americans and non-Hispanic whites.

Social support and social conflict. The roles of social relational factors such as support and conflict with friends, family (immediate and extended), work-related social networks, and other social groups (religious, volunteer, etc.) have also attracted attention from scholars in health and immigration literature. Cross-cultural findings suggest that Asian Americans are more likely than other racial/ethnic groups to engage in group recreation with friends and family (Kim and McKenry 2002). The emphasis on familial support and group solidarity in Chinese culture provides individuals with greater coping mechanisms for psychological distress, and cause lower comorbidity rates and older age of onset of depression (Goebert 2009; Hwu et al. 1996), which may also explain the lower prevalence of depression. However, the strong cultural emphasis on solidarity and interdependence among family and peers could also lead to the family and peer group placing too many demands on an individual, leaving them feeling compelled to fulfill obligations beyond their ability. While some studies found that a low level of perceived social support (Hwang et al. 2000; Gao et al. 2009) and a high level of social conflict (Ying and Han 2007; Zhang et al. 1997) were associated with higher depression risk, increasing evidence has suggested that the associations were more nuanced than previously thought (Greenberger and Chen 1996; Tsai and Levenson 1997).

Specifically, two major issues should be noted. First, as discussed earlier, different types of social relationship (immediate family, extended family, peers, work relationship, etc.) may vary in how they affect distress and depression. For example, a study that compared Chinese adolescents from Tianjin, China to US adolescents found that the strength of the effects of family cohesion varied by cultural setting, but the effect of perceived peer group warmth did not (Greenberger and Chen 1996). This suggests there are different mechanisms through which family and peer groups affect mental well-being. Data on a metropolitan sample of Chinese Americans showed a significant, negative association between depression and family support, and no significant effect of support from non-family members (Lieber et al. 2001). In addition, a study of Asian Americans also found discrepancies in the effects of familial or peer strains (Sangalang and Gee 2012). These findings indicates the necessity to examine relationships whether inside or outside of the familial setting, separately.

Second, researchers have noted that sociocultural factors and contexts could moderate the effects of social relationships on depression. Greenberger and Chen (Greenberger and Chen 1996) found that the quality of family relationships had a significantly stronger association with depression among the Asian American sample than among the European American sample. They pointed out that the cultural emphasis on respect and obedience for parents in Asian culture, and the later expected age of independence might explain such difference. However, in another comparative study between Chinese American college-aged individuals and non-Hispanic whites, researchers found no difference in the association between interpersonal conflict and depressive mood (Tsai and Levenson 1997).

In summary, literature suggests that it is necessary to examine social support and social conflict while distinguishing their sources (familial or peers) and control for the sociocultural contexts. However, the generalizability of the study results were limited because the study samples were adolescents or college-aged individuals (Greenberger and Chen 1996; Almeida et al. 2011), Asian Americans rather than Chinese Americans (Sangalang and Gee 2012), regional community data (Lieber et al. 2001), or the elderly (Wu et al. 2004). Furthermore, no study has compared the effects of 
the social relational factors on depression across different generations of Chinese Americans. Chinese Americans are likely, depending on their generational status, to differ both their adoption of American mainstream culture, retention of Chinese culture, and sociodemographic characteristics, all of which, according to previous literature, could moderate the effects of social relational factors on depression.

\section{Methods}

\subsection{Data Source}

This study uses publicly available data from the Collaborative Psychiatric Epidemiology Surveys (CPES) funded by the National Institute of Mental Health (NIMH). This survey joins together three nationally representative surveys: the National Comorbidity Survey Replication (NCS-R), the National Survey of American Life (NSAL), and the National Latino and Asian American Study (NLAAS). The CPES survey population includes adults age 18 and older, living in households in the 48 coterminous United States (NCS-R, NSAL), and the population for the Latino and Asian ancestry groups extends to the State of Hawaii (NLAAS). All three surveys were conducted between 2001 and 2003. Respondents in all three surveys were selected from a four-stage clustered area probability sample of households. Although each survey has unique features, they share a common core of measurements of primary mental health diagnostic symptoms, symptom severity, and mental health-related service utilization. More details on CPES sample design features are provided by Heeringa et al. (2004).

This study focuses on Chinese American respondents while using non-Hispanic white as a comparison group. The CPES data consist of 600 Chinese Americans from NLAAS, and 7587 non-Hispanic whites from NCS-R and NSAL. Specifically, the non-Hispanic white subsample from NSAL $(n=891)$ is not considered optimal for descriptive analysis of the white adult population, or comparative analyses between whites and non-black minority groups (Jackson et al. 2004), and is therefore dropped. Also excluded are 2516 non-Hispanic white cases from NCS-R Part I, who did not complete the more in-depth interview. The sampling design and interview schedule are detailed by Kessler and colleagues (Kessler et al. 2004). The procedure results in a sample of 4180 non-Hispanic white respondents from NCS-R. The sample used in the analyses after listwise deletion consists of 599 Chinese Americans and 3894 non-Hispanic whites.

\subsection{Measures}

Race/ethnicity and generation status. Race/ethnicity was defined using the self-identified primary racial/ethnic identity. Three generation groups were identified among Chinese Americans: foreign-born to foreign-born parents (1st generation), US-born to at least one foreign-born parent (2nd generation), and US-born adults to US-born parents (3rd-or-higher generations).

Diagnostic assessment. Prevalence of major depressive disorder in the past twelve months was measured using the World Mental Health Survey Initiative Version of the World Health Organization Composite International Diagnostic Interview (WMH-CIDI), a fully structured lay-administrated diagnostic instrument that generates both DSM-IV and International Classification of Diseases, 10th Revision diagnoses (Kessler et al. 2004). The WMH-CIDI was validated for the US and Chinese populations (Kessler and Üstün 2004). The DSM-IV diagnosis of major depressive disorder (MDD) is used in this study. To assess depressive symptoms, CIDI included twenty-five questions which could be combined into indicators of nine specific symptoms, including depressed mood, decreased interest or pleasure, change in weight or appetite, change in sleep, change in activity, fatigue, guilt, concentration, and suicidality.

Chronic physical condition. Respondents were asked if they ever had chronic physical conditions from a standard chronic disorder checklist used in the US National Health Interview Survey. Conditions include cancer, frequent or severe headache, heart disease, high blood pressure, asthma, ulcer, or stroke. Although prior research has found differential associations between various chronic conditions and depression, the purpose of this study is to examine how the presentence of any chronic physical 
conditions was related to depression. Therefore, a dichotomous variable was created. Respondents who had at least one of the six conditions were coded as "yes", and those who did not were coded as "no." Prior research has demonstrated reasonable accuracy of such self-reported measures (Kriegsman et al. 1996). This dichotomous variable has previously been found to be a valid predictor depression among Chinese populations (Lee et al. 2009).

Social support and conflicts. The friend support scale and the relative support scale measure the perceived level of support from the two domains. The friend support scale was measured on a scale consisting of three items on how often respondents talk on the phone, get together with relatives, and how often they can rely on relatives to discuss worries (Cronbach's alpha $=0.77$ ). The answers were "not at all" "little" "some" or "a lot." The relative support scale consisted of three parallel items (Cronbach's alpha $=0.70)$. For both scales, a higher numeric value indicates a higher level of support. Conflict with friends (Cronbach's alpha $=0.60)$ and relatives (Cronbach's alpha $=0.70)$ was assessed separately, each with two questions, specifically, how often friends or relatives made too many demands of respondents, or argue with, respondents. The answers were "never" "rarely" "some" or "often." A higher numeric value represented a higher level of conflict. Researchers have previously reported moderate to high reliability (Cronbach's alpha ranging from 0.59 to 0.71 ) of the four scales (Sangalang and Gee 2012; Nicdao et al. 2008; Mulvaney-Day et al. 2007; Canino et al. 2008). Studies have found these measures to be significant predictors of mental health service use or depressive disorder among Asian populations (Sangalang and Gee 2012; Nicdao et al. 2008). In the present study, scales are normalized based on the mean and standard deviation values of the study sample.

Sociodemographic characteristics. In regard to the measurement of race/ethnicity, when a respondent identifies with multiple race/ethnicity categories, he or she was assigned to a single category according to priority order in the NSAL and NLAAS respondent classification rules (e.g., Vietnamese over Chinese). Other sociodemographic correlates include gender, age in years, marital status, having a college degree, work status, and poverty level. Specifically, marital status was coded in three categories, never married, married/cohabiting, and divorced/separated/widowed. Poverty level was calculated as the ratio of the household income to the 2001 US Department of Health and Human Services poverty guideline, adjusting for number of persons in the household.

\subsection{Statistical Analysis}

All statistical analyses were conducted using Stata 14 (StataCorp 2015). CPES provided weights that should be applied in computing sampling errors for sample estimation of descriptive statistics and regression parameters. The CPES weighting computation was designed based on the geographic domains of the US national sample frame used in the clustered sampling, and the race/ethnicity of the respondent (CPES 2007; CPES 2008). Binary logistic regression was used to study correlates of MDD. Multivariate significance tests were calculated using Wald tests based on coefficient variance-covariance matrices that were adjusted for design effects using the Taylor's series linearization method. Because sampling weights were used in the analyses, traditional tests for goodness of fit could not be used. It is worth noting that to my best knowledge, there is currently no goodness-of-fit test for binary logistic regression analysis of complex survey data involving subpopulation estimation, hence my inability to evaluate the predictive power of the models in the multivariate analyses.

\section{Results}

Table 1 presents the weighted descriptive statistics of the study sample, separately for Chinese Americans (CAs) in three generational groups, and non-Hispanic whites (NHWs). There is a great generational difference of MDD prevalence among CAs, with first generations having the lowest prevalence $(3.71 \%)$, which is significantly lower than that among second generation CAs $(9.08 \%)$ and NHWs $(7.26 \%)(p<0.05)$. Although second generation CAs have the highest prevalence, it was not statistically different from that among third-or-higher generation CAs (6.58\%) and NHWs (Figure 1). Second generation CAs $(40.64 \%)$ are also much more likely than first $(15.92 \%)$ or third-or-higher 
(28.20\%) generation CAs and non-Hispanic whites (19.43\%) to never be married. The proportion of the college educated is much higher among CAs, especially second generations $(55.88 \%)$, when compared to NHWs $(26.84 \%)$. There is greater household income heterogeneity among CAs than NHWs; CAs, regardless of generational status, are more likely to live under the poverty line and more likely to have a household income 6 or more times of poverty line, than did NHWs. There are also significant differences in gender composition and work status among the subsamples.

Table 1. Weighted Descriptive Statistics of Chinese Americans and Non-Hispanic Whites.

\begin{tabular}{|c|c|c|c|c|}
\hline & $\begin{array}{c}\text { 1st G CA } \\
\% \text { or mean (s.e.) }\end{array}$ & $\begin{array}{c}\text { 2nd G CA } \\
\% \text { or mean (s.e.) }\end{array}$ & $\begin{array}{l}\text { 3rd-or-higher G CA } \\
\text { \% or mean (s.e.) }\end{array}$ & $\begin{array}{l}\text { Non-Hispanic White } \\
\% \text { or mean (s.e.) }\end{array}$ \\
\hline$n$ & 475 & 74 & 50 & 3892 \\
\hline DSM-IV MDD in 12 months & $3.71 \%(0.01)$ & $9.08 \%(0.03)$ & $6.58 \%(0.03)$ & $7.26 \%(0.003)$ \\
\hline female & $54.20 \%(0.02)$ & $56.47 \%(0.07)$ & $41.84 \%(0.05)$ & $51.84 \%(0.01)$ \\
\hline age (mean) & $43.67(1.25)$ & $39.05(2.89)$ & $41.19(1.61)$ & $46.70(0.56)$ \\
\hline \multicolumn{5}{|l|}{ marital status } \\
\hline never married & $15.92 \%(0.02)$ & $40.64 \%(0.09)$ & $28.20 \%(0.06)$ & $19.43 \%(0.01)$ \\
\hline married/cohabiting & $74.16 \%(0.02)$ & $45.19 \%(0.08)$ & $50.63 \%(0.12)$ & $61.13 \%(0.01)$ \\
\hline divorced/separated/widowed & $9.92 \%(0.02)$ & $14.17 \%(0.05)$ & $21.18 \%(0.12)$ & $19.44 \%(0.01)$ \\
\hline \multicolumn{5}{|l|}{ family income level } \\
\hline below poverty line & $21.47 \%(0.03)$ & $17.52 \%(0.05)$ & $16.19 \%(0.06)$ & $8.14 \%(0.01)$ \\
\hline $1-3$ times of poverty line & $16.88 \%(0.02)$ & $15.56 \%(0.06)$ & $12.29 \%(0.04)$ & $25.44 \%(0.01)$ \\
\hline 3-6 times of poverty line & $23.18 \%(0.02)$ & $23.24 \%(0.06)$ & $21.45 \%(0.13)$ & $33.04 \%(0.01)$ \\
\hline$\geq 6$ times of poverty line & $38.47 \%(0.04)$ & $43.68 \%(0.06)$ & $50.07 \%(0.17)$ & $33.38 \%(0.02)$ \\
\hline \multicolumn{5}{|l|}{ work status } \\
\hline employed & $65.08 \%(0.03)$ & $57.81 \%(0.06)$ & $68.78 \%(0.12)$ & $65.95 \%(0.01)$ \\
\hline unemployed & $5.28 \%(0.01)$ & $4.42 \%(0.02)$ & $10.97 \%(0.09)$ & $5.07 \%(0.01)$ \\
\hline not in labor force & $29.65 \%(0.03)$ & $37.77 \%(0.06)$ & $20.25 \%(0.07)$ & $28.98 \%(0.01)$ \\
\hline chronic physical condition & $27.05 \%(0.03)$ & $33.40 \%(0.07)$ & $47.36 \%(0.08)$ & $50.19 \%(0.01)$ \\
\hline friend support $(z)$ & $-0.40(0.06)$ & $0.30(0.09)$ & $0.31(0.14)$ & $0.29(0.02)$ \\
\hline relative support $(z)$ & $-0.54(0.06)$ & $0.07(0.15)$ & $0.02(0.10)$ & $0.14(0.02)$ \\
\hline friend conflict $(z)$ & $-0.02(0.06)$ & $0.31(0.10)$ & $0.25(0.10)$ & $-0.03(0.02)$ \\
\hline relative conflict ( $(z)$ & $-0.06(0.05)$ & $0.17(0.13)$ & $0.15(0.13)$ & $-0.13(0.02)$ \\
\hline$N=4491$ & & & & \\
\hline
\end{tabular}

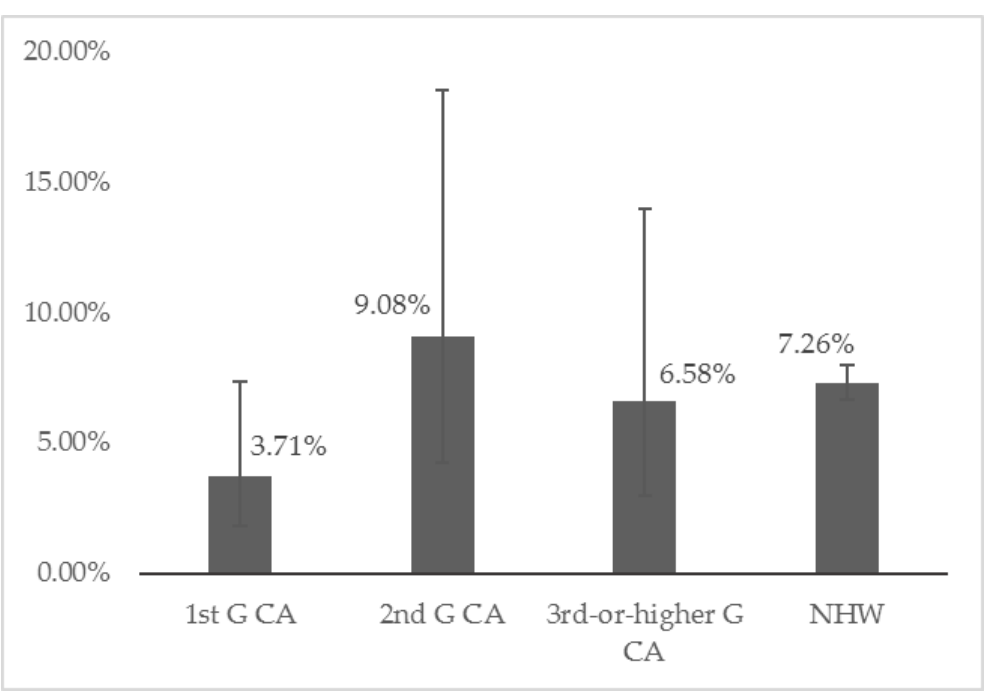

Figure 1. Weighted Prevalence of 12-Month MDD.

Regarding the main predictors of interest, the prevalence of any chronic physical conditions is much lower among first $(27.05 \%)$ and second (33.40\%) generation CAs than among NHWs $(50.19 \%)$, and the prevalence is particularly low among first. Social support from friends is distinctively lower among first generation CAs $(-0.40)$ than second (0.30) or third-or-higher $(0.31)$ generation CAs 
and NHWs (0.29). Support from relatives is generally lower among CAs, particularly so for first generations (-0.54), than for NHWs (0.14). Interestingly, second and third-or-higher generation CAs report higher levels of conflicts with friends ( 0.31 and 0.25 , respectively) and relatives ( 0.17 and 0.15 , respectively), while first generation CAs have relatively similar levels of conflicts with friends $(-0.02)$ and relatives $(-0.06)$ with non-Hispanic whites $(-0.03$ for friend conflict, and -0.13 for relative conflict). In summary, there are significant differences in the main predictors, the outcome variable, and several sociodemographic factors among different generations of CAs and NHWs.

Nested multivariable logistic regression is used to examine the correlates of depression, the weighted results presented in Table 2. In Model 1, I compare three generational groups of CAs to NHWs while controlling for sociodemographic factors. The regression coefficients show that only first generation CAs are significantly different from the reference group, NHWs $(\beta=-0.81, p<0.05)$. Being female, never married, and not in labor force are associated with a higher likelihood of having MDD in the past year. Age has a curvilinear relationship with depression, with middle age (approximately 40 ) having the highest predicted probability of depression.

Table 2. Weighted Logistic Regression Results of Twelve-Month DSM-IV MDD.

\begin{tabular}{|c|c|c|c|c|}
\hline & Model 1 & Model 2 & Model 3 & Model 4 \\
\hline NHW (ref) & 1 & 1 & 1 & 1 \\
\hline 1st G CA & $-0.81(0.40)^{*}$ & $-0.83(0.41) *$ & $-0.86(0.42) *$ & $-0.71(0.42) *$ \\
\hline 2nd G CA & $0.05(0.44)$ & $0.04(0.42)$ & $-1.24(1.14)$ & $-0.23(1.14)$ \\
\hline 3rd-or-higher G CA & $-0.39(0.35)$ & $-0.41(0.37)$ & $0.73(0.68)$ & $-0.77(0.68)$ \\
\hline chronic physical & condition & $0.96(0.14)^{* * *}$ & $0.95(0.15)^{* * *}$ & $0.97(0.14)^{* * *}$ \\
\hline \multicolumn{2}{|l|}{ 1st G CA * chronic } & & $0.44(0.45)$ & \\
\hline \multicolumn{2}{|c|}{ 2nd G CA * chronic } & & $0.90(1.07)$ & \\
\hline \multicolumn{2}{|c|}{ 3rd-or-higher G CA * chronic } & & $-2.89(2.34)$ & \\
\hline \multicolumn{2}{|c|}{ FS } & $-0.21(0.06)^{* * *}$ & $-0.20(0.07)^{* *}$ & $-0.30(0.09) * *$ \\
\hline \multicolumn{2}{|l|}{ RS } & $-0.26(0.09) * *$ & $-0.30(0.09) * *$ & $-0.21(0.06)^{* *}$ \\
\hline \multicolumn{2}{|l|}{ FC } & $0.13(0.06) *$ & $0.20(0.07) * *$ & $0.20(0.07) * *$ \\
\hline \multicolumn{2}{|l|}{$\mathrm{RC}$} & $0.18(0.07) *$ & $0.12(0.07)$ & $0.12(0.07)+$ \\
\hline \multicolumn{2}{|l|}{1 st G CA * FS } & & $0.68(0.40) \dagger$ & $0.70(0.39)+$ \\
\hline \multicolumn{2}{|l|}{ 2nd G CA * FS } & & $1.03(0.58) \dagger$ & $0.72(0.42)+$ \\
\hline \multicolumn{2}{|l|}{ 3rd-or-higher G CA * FS } & & $-0.62(1.68)$ & $-0.27(0.87)$ \\
\hline \multicolumn{2}{|l|}{1 st $G C A * R S$} & & $0.07(0.17)$ & \\
\hline \multicolumn{2}{|l|}{2 nd G CA * RS } & & $-0.78(0.54)$ & \\
\hline \multicolumn{2}{|l|}{ 3rd-or-higher G CA * RS } & & $-0.33(0.81)$ & \\
\hline \multicolumn{2}{|l|}{ 1st $\mathrm{GCA} * \mathrm{FC}$} & & $-0.60(0.30) *$ & $-0.62(0.30) *$ \\
\hline \multicolumn{2}{|l|}{ 2nd G CA * FC } & & $-1.01(0.38) * *$ & $-0.89(0.48) \dagger$ \\
\hline \multicolumn{2}{|l|}{ 3rd-or-higher G CA * FC } & & $0.30(0.55)$ & $0.61(0.62)$ \\
\hline \multicolumn{2}{|l|}{ 1st $\mathrm{GCA}^{*} \mathrm{RC}$} & & $0.29(0.33)$ & $0.28(0.32)$ \\
\hline \multicolumn{2}{|l|}{ 2nd G CA * RC } & & $1.07(0.40) * *$ & $0.65(0.43)$ \\
\hline \multicolumn{2}{|l|}{ 3rd-or-higher G CA * RC } & & $-1.05(1.45)$ & $-1.23(0.79)$ \\
\hline female (ref: male) & $0.65(0.08) * * *$ & $0.72(0.08) * * *$ & $0.71(0.08)^{* * *}$ & $0.72(0.08)^{* * *}$ \\
\hline age & $0.11(0.03) * * *$ & $0.08(0.03)^{* * *}$ & $0.08(0.03) * *$ & $0.08(0.03) * *$ \\
\hline age^2 & $-0.001(0.0003) * * *$ & $-0.001(0.0003) * * *$ & $-0.001(0.0003) * * *$ & $-0.001(.0003) * * *$ \\
\hline never married (ref) & 1 & 1 & 1 & 1 \\
\hline married/cohabitating & $-0.68(0.18) * * *$ & $-0.75(0.18) * * *$ & $-0.73(0.18)^{* * *}$ & $-0.73(0.18)^{* * *}$ \\
\hline $\operatorname{div} / \operatorname{sep} /$ wid & $0.29(0.20)$ & $0.17(0.20)$ & $0.19(0.20)$ & $0.17(0.20)$ \\
\hline employed (ref) & 1 & 1 & 1 & 1 \\
\hline unemployed & $0.49(0.28)$ & $0.41(0.29)$ & $0.36(0.30)$ & $0.36(0.30)$ \\
\hline not in labor force & $0.68(0.17)^{* * *}$ & $0.56(0.17)^{* * *}$ & $0.54(0.17)^{* *}$ & $0.53(0.17)^{* *}$ \\
\hline college & $-0.06(0.13)$ & $0.12(0.13)$ & $0.11(0.13)$ & $0.12(0.13)$ \\
\hline $\begin{array}{l}\text { constant } \\
N=4491\end{array}$ & $-4.27(0.50)$ & $-4.13(0.53)$ & $-4.11(0.54)$ & $-4.12(0.53)$ \\
\hline
\end{tabular}

In Model 2, I included the five predictors of MDD, chronic physical condition, friend support, relative support, friend conflict, and relative conflict. First generation CAs are still less likely to report depression than do non-Hispanic whites $(\beta=-0.83, p<0.05)$. There are no major changes in the coefficients for the sociodemographic factors included. 
In Model 3 I included the interaction terms between generational status and chronic physical condition, and between generational status and each of the four social relational factors. The coefficients for demographic and socioeconomic factors only changed slightly in magnitude. The main effect of being first generation $C A$ is statistically significant $(\beta=-0.86, p<0.05)$. The main effects of having any chronic physical condition $(\beta=0.95, p<0.001)$, friend support $(\beta=-0.20, p<0.01)$, relative support $(\beta=-0.30, p<0.01)$, friend conflict $(\beta=0.20, p<0.05)$ are significant, but the main effect of relative conflict is not. Friend conflict and relative conflict both have significant interaction effects with generational status. None of the interaction terms for relative support and chronic physical condition are significant. Therefore, I exclude them in the next step of analysis.

In Model 4, only the three sets of interaction terms that have shown significance in Model 3 are included, specifically, the interaction terms between the three generational groups and friend support, friend conflict, and relative conflict. The main effect of being first generation CAs, as compared to non-Hispanic whites, is significant $(\beta=-0.71, p<0.05)$. The interaction term "1st G CA $\times$ friend support" $(\beta=0.70, p<0.10)$ and "2nd G CA $\times$ friend support" $(\beta=0.72, p<0.10)$ are marginally significant.

Figure 2 presents the predicted probability of MDD by level of friend support for all four groups, with other variables held at sample mean. For the NHWs, a higher level of friend support is associated with a lower likelihood for MDD. The slopes for first and second G CAs are positive, but only marginally significant. Figure 2 also demonstrates that the generational differences of depression risks vary by the level of friend support. Among those with a standardized friend support score lower than $1.01^{1}$, first generation CAs are less likely than NHWs to have MDD, with other variables held constant; among those with a standardized friend support score higher than 1.01, first generation CAs are more likely that non-Hispanic whites to have MDD. The two groups have the same predicted probability of having MDD when their standardized friend support score is 1.01, which is approximately one standard deviation above the sample average of friend support. The main effect of being second generation CAs (vs. NHWs) is not significant. The interaction term terms "2nd G CA $\times$ friend support" $(\beta=0.72, p<0.10)$ is marginally significant. These results indicate whether second generation CAs are more than, less than, or equally as likely as NHWs to have MDD in the past year is contingent on the levels of friend support.

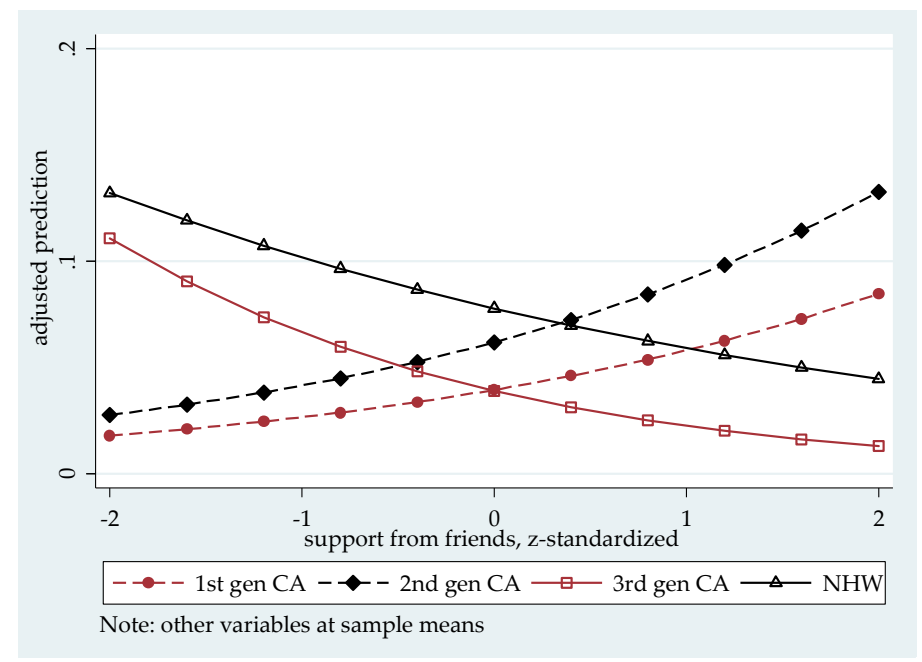

Figure 2. Predicted Probability of 12-month MDD by Friend Support.

$1-(-0.71) / 0.70=1.01$, which is -1 times the coefficient for the main effect of being first generation CA divided by the coefficient for the interaction term "1st G CAs $\times$ FS." 
Figure 3 presents the predicted probability of MDD by level of friend conflict for all four groups, with other variables held at sample mean. The effect of friend conflict for first generation CAs is significantly different from that for NHWs. While the slope for NHWs is significant and positive, it is negative for first generation CAs, which means that a higher level of friend conflict is associated with a lower risk for MDD among first generation CAs. Friend conflict is also negatively associated with MDD risk for second generation CAs, but adjusted Wald test find this slope only marginally significant $(p<0$.10). Furthermore, Figure 3 demonstrates that the difference between first generation CAs and NHWs vary by the levels of friend conflict. Among those with a standardized friend conflict score lower than $-1.14^{2}$, first generation CAs are more likely than non-Hispanic whites to have MDD; among those with a standardized friend conflict score higher than -1.14 , first generation CAs with are less likely than non-Hispanic whites to have MDD. The two groups have the same predicted probability of having MDD when their standardized friend conflict score is -1.14 , which is slightly more than one standard deviation below the sample average friend conflict level.

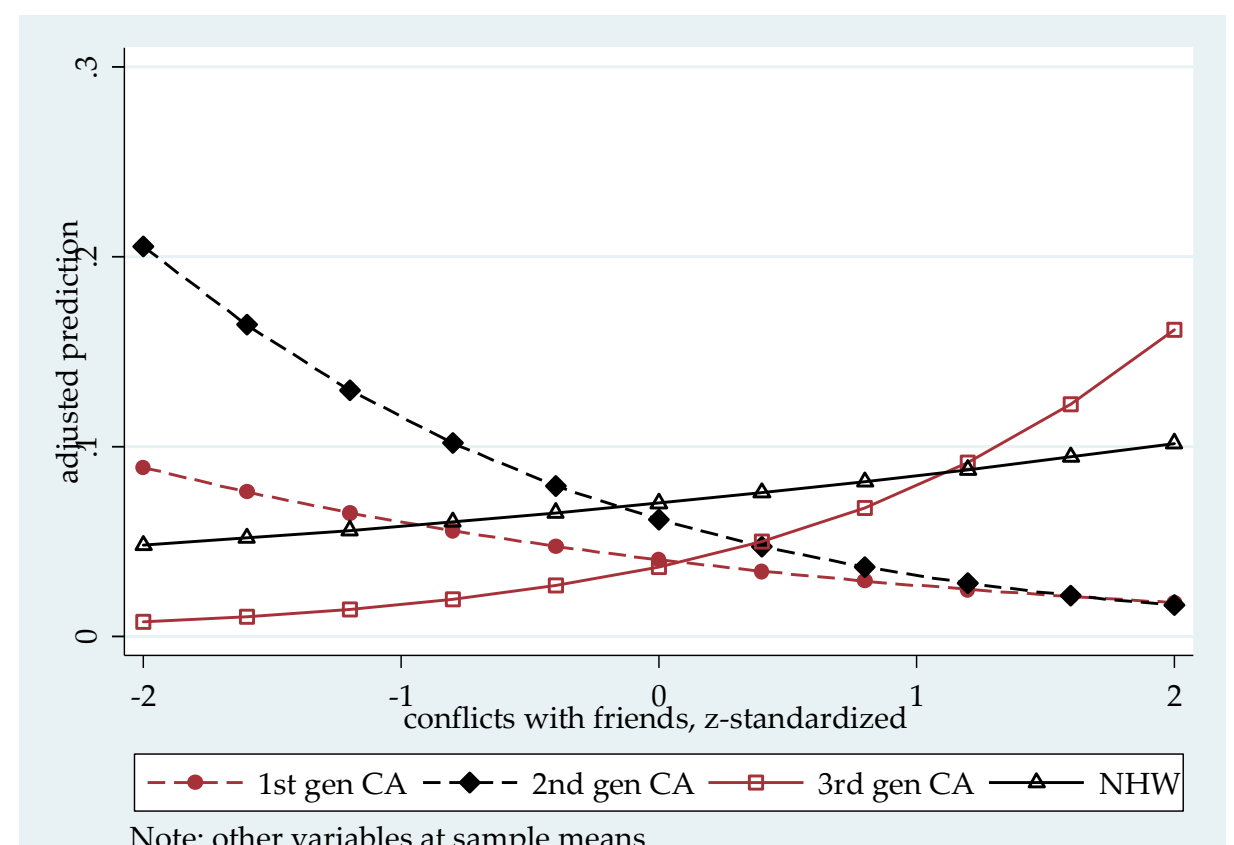

Figure 3. Predicted Probability of 12-month MDD by Friend Conflict.

Among those with a standardized friend conflict score of zero (sample average), second generation CAs do not differ significantly from NHWs with the same level of friend and relative conflicts on MDD risk, with other variables held constant. Among those with a standardized friend conflict score higher than $-0.26^{3}$, second generation CAs are less likely than NHWs to have MDD, with other variables held constant; among those with a standardized friend conflict score lower than -0.26 , second generation CAs have a higher MDD risk than that among NHWs. The difference in MDD risk between second generation CAs and NHWs is bigger when their level of friend conflict is either very high or very low.

The main effect of being third-or-higher generation CAs (vs NHWs) is not statistically significant, and none of its interaction terms is significant, which means that third-or-higher generation CAs are

$2-(-0.71) /-0.62=-1.14$, which is -1 times the coefficient for the main effect of being first generation CA divided by the coefficient for the interaction term " 1 st G CAs $\times$ FC."

$3-(-0.23) /-0.89=-0.26$, which is -1 times the coefficient for the main effect of being second generation CAs divided by the coefficient for the interaction term "2nd G CAs $\times$ FC." 
not significantly different from the NHWs on MDD risk, regardless of the level of friend support, friend conflict, or relative conflict.

The main effect of relative support is significant and negative $(\beta=-0.21, p<0.01)$, which indicates that for all CAs and NHWs, a higher level of relative support is associated with a lower likelihood of having MDD in the past twelve months. The main effect of relative conflict is only marginally significant $(\beta=0.12, p<0.10$ ), which indicates a positive association between relative conflict and MDD risk for the reference group, non-Hispanic whites. None of the interaction terms between relative conflict and generational status is significant, indicating the effect of relative conflict have the same direction and magnitude for all four groups.

Having any chronic physical conditions is still positively associated with the likelihood of depression $(\beta=0.95, p<0.001)$. It indicates that for all groups, having at least one chronic physical condition increases the likelihood of having MDD.

Figure 4 shows the predicted probability of MDD for all four groups, with the covariates held at their sample means. Among CAs, second generation have the highest predicted probability $(6.21 \%)$ of having MDD, compared to first (4.05\%) and third-or-higher (3.60\%) generation CAs. Generational differences notwithstanding, CAs in general have lower predicted probabilities of having MDD than did NHWs (6.99\%).

Among the sociodemographic variables included, women (vs. men), being never married (vs. married or cohabitating), and not being in the labor force (vs. employed) are associated with elevated likelihoods of having MDD in the past year, with other covariates held constant. Additionally, age has a curvilinear relationship with depression, with the likelihood of having MDD peaking around the age of 35, and decreasing on both ends (predicted probability of MDD by age available upon request).

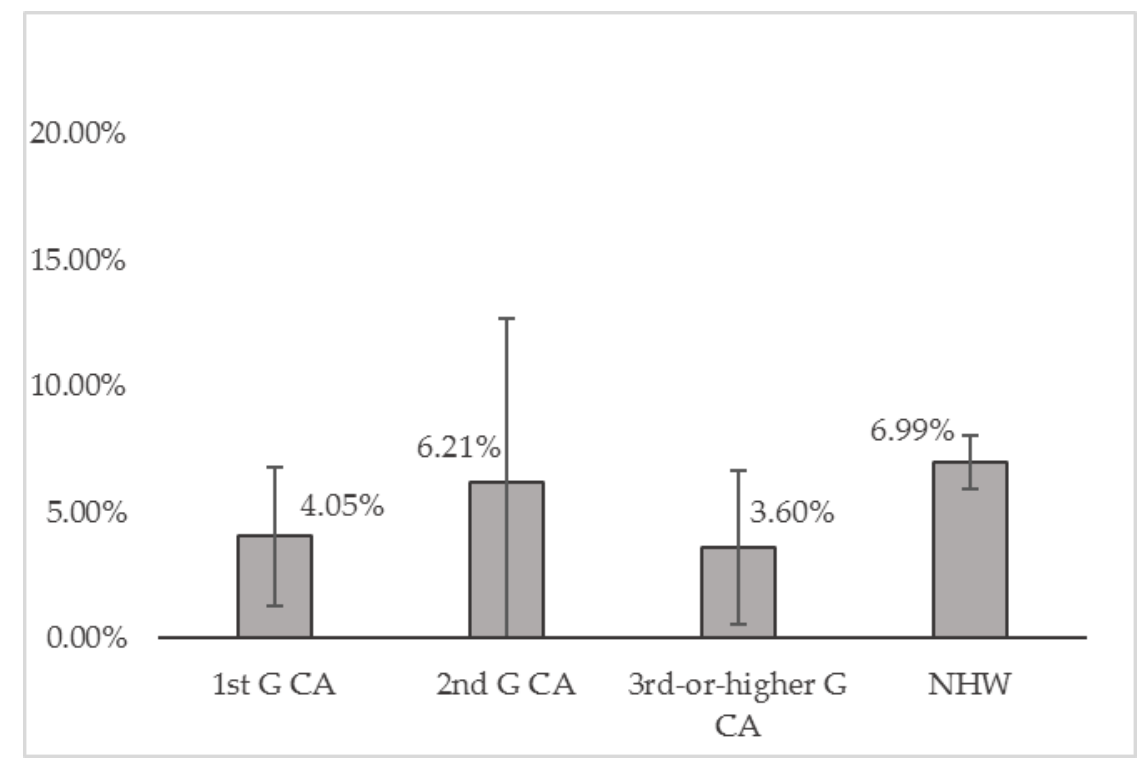

Figure 4. Predicted Probability of MDD in Past Year.

\section{Discussion}

This study has several important findings on the generational differences of depression among Chinese Americans, and correlates of depression for each generation of Chinese Americans. First, with sociodemographic and relational factors controlled for, there are significant difference in depression risks among different generations of Chinese Americans, as shown in Figure 4. Two possible explanations are offered here. One the one hand, the drastically higher predicted probability of MDD for second generation Chinese Americans is in line with previous findings. Specifically, research shows that second generation immigrants are "between two worlds," that they have to negotiate, 
and address the conflicts between, two sets of cultural values presented by their foreign-born parents and their (mostly) US-born peers (Zhou 1997; Das and Kemp 1997), which is distressing. In comparison, first generation Chinese Americans, with, in general, a stronger subscription to Chinese cultural values, may feel less compelled to assimilate to US values, hence the lower risks for MDD. On the other hand, cultural psychiatrists have argued that cultural factors have significant influence on the way people experience and express impaired mood (Kleinman 1982; Marsella 1978). For example, studies have noted a tendency among the Chinese populations to somatize their psychological distress (Kleinman 1977; Mezzich et al. 1999), and suppress emotional/affective symptoms (Tseng and Hsu 1970). Using a universal measurement or criterial to diagnose depression could lead to inaccurate estimation of the prevalence rates (Lin 1989). Therefore, it is possible that first generation Chinese Americans, under relatively heavier influence of Chinese cultural values, express distress in a different way to other Chinese Americans and non-Hispanic whites, to the degree that the some of their symptoms were not captured by DSM. Such theories are yet to be fully tested in empirical studies.

Neither the descriptive analysis nor the multivariate logistic regression analysis showed any significant difference between third-or-higher generation Chinese Americans and non-Hispanic whites. Even though the predicted MDD risk of the former (3.60\%) was only slightly more than half of the latter $(6.99 \%)$ (shown in Figure 4$)$, the difference between two percentages was not statistically significant. The regression coefficients reported no statistical significance among the slopes either. Such results might be due to the small sample size of the former group $(n=50)$. If this drastic discrepancy between the two groups, albeit non-significant, did suggest a tendency of generalizable difference, it could only be explained by factors not examined in the analyses of this study, factors with protective mental health effects for third-or-higher generation Chinese Americans that could explain the much lower predicted probability. The existing body of literature has no suggestions on what these factors could be.

The lower risks of depression among first generation Chinese Americans also indicates that in addition to the factors examined in this study, there are other factors that would explain the ethnic disparities of depression between first generation Chinese Americans and non-Hispanic whites. It could be the case that Chinese Americans benefited from the protective effects of certain factors that would help to avoid and/or alleviate distress. Previous studies have suggested that strong family cohesion is an important norm in Chinese culture that helps individuals coping with difficult situations (Salant and Lauderdale 2003; Juang and Alvarez 2010). It is possible that a sense of familial belongingness and support from immediate family members protects Chinese Americans from distress or helps them better cope with it. However, the lack of data on family support among non-Hispanic whites limited my ability to test this possible explanation. Future research should explore the association between family-related factors and depression among Chinese Americans.

In addition, this study found that friend conflict is negatively associated with depression among first generation Chinese Americans. This finding seems to challenge the general understanding that social conflicts tend to cause distress which may lead to depression. However, the cross-sectional nature of the data in this study invites interpretation from another causal direction. It could be the case that first generation Chinese Americans who were suffering from depression were well supported by friends, that these friends may actively avoid making too much demands, or arguing with them. Future research should test this hypothesis using qualitative methods or longitudinal data.

This explanation is even more convincing given the marginally significant positive association between friend support and depression risk among first generation Chinese Americans, another finding that seems puzzling at first, but could also be interpreted as showing this network's greater willingness to offer support to those who were depressed. For first generation immigrants, a network of friends is the most feasible social network for an individual to establish, when compared to the difficulties presented by helping family migrate from their countries of origin, or even creating new family (through marriage). For example, a study of Asian immigrant young adults found friend support to be a protective factor against the effects of family conflict on depression, but only among first-generation immigrants (Obradović et al. 2013). Findings of this study expanded the existing body 
of literature by showing a nuanced, two-way relationship between support and conflict from friends and depression. In addition, my study findings suggest that it is more likely that friendship networks serve as a source of coping for first-generation Chinese Americans who were already depressed, given a higher risk of depression is associated with greater friend support and less friend conflict. In other words, the main causes of depression for this group are likely to be sources other than friendship networks; instead of causing distress, friends of first generation Chinese Americans more supportive and less confrontational or demanding when faced with a first generation Chinese American suffering from depression.

Furthermore, the negative association between relative support and depression risk among first generation Chinese Americans could mean that extended family provided the support needed to decrease the risk of depression. The significant effects of friend support and relative support, along with the marginally significant effect of friend conflict, provided a complicated picture of the roles of social networks in affecting the mental well-being of first generation Chinese Americans. Networks of friends help reduce stress and provide an increased level of solace and support (through decreases in conflict), while familial networks provide emotional support that also help reduce the risk of depression. If true, the effect of relative support on depression would have important implications for involving both friend and familial networks in depression prevention and treatment programs. Future research is needed to confirm the differential effects of social relational factors for different generations of Chinese Americans.

Similar to the first generation Chinese Americans, second generation Chinese Americans saw a higher likelihood of depression associated with a higher level of friend support (Figure 2), and a lower level of friend conflicts (Figure 3). One explanation is that for second generation Chinese Americans, friendship was a particularly important social network with high efficacy, such that friends were unlikely to argue with individuals suffering from depression. The second generation Chinese American sample reported the highest level of friend conflicts, which provided further context for this explanation: although second generation Chinese Americans generally have a high level of conflicts with friends, they are well supported when they are in poor mental health. Social network studies among immigrants found that for second generation immigrants, peer group is also the most important form of social network; the lack of strong cultural belonging to, or worse, the sense of alienation from either ethnic or the mainstream culture tend to push second generation immigrants to form strong bonds with people who either shared, or understood, their experience (Portes and Rumbaut 2001).

This study is not without limitations. First and foremost, the use of cross-sectional survey data limited my ability to make any causal inference, hence unspecified directionality of the influences among depression, socio-demographic characteristics, chronic physical condition, and social support and conflicts. Experimental and longitudinal research effort is need to better understand how social support and conflicts interact with depression risk. Also, the author was not able to include several important factors, including perceived discrimination, family cohesion, and family conflicts in my analyses because of the lack of data on these measures from the non-Hispanic white sample. Previous studies have found the effects of these factors on mental well-being among Asian American populations to be significant yet nuanced, and the findings were less than consistent for the potential reasons regarding sampling frames and strategies (Gee et al. 2007; Juang et al. 2007). It should also be noted that over three quarters of the Chinese Americans in the study sample were first generation immigrants, which has skewed the findings among second and third-or-higher generation Chinese Americans. Future research should try and replicate the finding of this study, using a sample with more balanced generation composition.

Furthermore, research has found variation in the patterns of mental disorders within the group of Asian or Chinese Americans. For example, Takeuchi and colleagues (Takeuchi et al. 2007) found that among foreign-born first generation Asian immigrants, those who arrived earlier in life were at higher risk of lifetime and twelve-month mental disorder, than those who arrived as an adult. Scholars have proposed the concept of "1.5 generation" immigrants, which refers to individuals who 
migrated during their childhood or middle to late adolescence (Park 1999; Rumbaut 1994). Compared to those who migrated to a new country as an adult, the 1.5 generation immigrants tend to have different experiences and unique adaptation strategies to the process of acculturation, compared to first generation immigrants, or US-born second generation immigrants (Bartley and Spoonley 2008; Kim et al. 2003; Lee 2011). One study found significant association between adult subjective social status and depression among Asian immigrants who came to the US when they were twenty-five years old or older, not among those who migrated before the age of twenty-five (Leu et al. 2008). Future comparative studies need to examine the depression patterns and correlates among the 1.5 generations, in comparison to other generations.

In addition, since one purpose of this study is to test the relationship between physical condition and mental status across different groups, chronic physical condition is coded into a dichotomous variable. In the future, examining how different types of physical conditions such as cancer, heart disease, or hypertension are associated with depression would help us better understand the etiology and effects of depression, and provide implications for disease prevention and management. Last but not least, the non-Hispanic white is not a homogeneous group. Yet, because of the lack of data on immigration related factors, the author was unable to take into account the potential variation in generational status, English proficiency, and other acculturation indicators of this group.

\section{Conclusions}

The present study examined depression prevalence and correlates among different generations of Chinese Americans, using non-Hispanic whites as a comparison group. Results of the study revealed that Chinese Americans in general have a lower risk of depression than non-Hispanic whites. Moreover, the prevalence and correlates of depression do not show a linear trend of difference from first to second to third-or-higher generation Chinese Americans, and then to non-Hispanic whites; rather, the risk of depression and its association with social relational factors presents in distinctive patterns for first and second generation Chinese Americans, compared to third-or-higher generation Chinese Americans and non-Hispanic whites. Specifically, friend and family network play different roles in their influence on depression risk for different generations of Chinese Americans. The findings of this study contributed to the growing body of literature on acculturation and mental health among immigrants, shedding light on the complicated sociocultural contexts that could influence the mental well-being of individuals. Mental health service providers need to be aware of the complex and nuanced association between social relational factors and depression in their prevention, management, and treatment efforts. Researchers need to further examine the causality issues, and the impacts of other sociocultural factors such as perceived discrimination, family cohesion, and acculturative stress among Chinese Americans.

Acknowledgments: The author thanks Gretchen A. Condran, Robert L. Kaufman, Shanyang Zhao, and Grace X. Ma, for their helpful comments and suggestions on earlier drafts of this paper. The author also thanks Andrew Turner and Amanda J. Turner who assisted in the language editing and proof-reading of the manuscript.

Conflicts of Interest: The author declares no conflict of interest.

\section{References}

Almeida, Joanna, S. V. Subramanian, Ichiro Kawachi, and Beth E. Molnar. 2011. Is Blood Thicker Than Water? Social Support, Depression and the Modifying Role of Ethnicity/Nativity Status. Journal of Epidemiology and Community Health 65: 51-56. [CrossRef] [PubMed]

Bartley, Allen, and Paul Spoonley. 2008. Intergenerational Transnationalism: 1.5 Generation Asian Migrants in New Zealand. International Migration 46: 63-84. [CrossRef]

Berry, John W. 1990. Acculturation and Adaptation: A General Framework. In Mental Health of Immigrants and Refugees. Edited by Wayne H. Holtzman and Thomas H. Bornemann. Austin: Hogg Foundation for Mental Health, pp. 90-102. 
Berry, John W. 2003. Conceptual Approaches to Acculturation. In Acculturation: Advances in Theory, Measurement, and Applied Research. Edited by K. M. Chun, P. Balls Organista and G. Marín. Washington: American Psychological Association, pp. 17-37.

Canino, Glorisa, William A. Vega, William M. Sribney, Lynn A. Warner, and Margarita Alegría. 2008. Social Relationships, Social Assimilation, and Substance Use Disorders among Adult Latinos in the U.S. Journal of Drug Issues 38: 69-101. [CrossRef] [PubMed]

Chen, Irene G., Robert E. Roberts, and Lu A. Aday. 1998. Ethnicity and Adolescent Depression: The Case of Chinese Americans. Journal of Nervous 186: 623-30. [CrossRef]

Cheung, Chau-Kiu, and Christopher Bagley. 1998. Validating An American Scale in Hong Kong: The Center for Epidemiological Studies Depression Scale (CES-D). Journal of Psychology 132: 169. [CrossRef] [PubMed]

Chou, Kee-Lee, Iris Chi, and Nelson W. S. Chow. 2004. Sources of Income and Depression in Elderly Hong Kong Chinese: Mediating and Moderating Effects of Social Support and Financial Strain. Aging \& Mental Health 8: 212-21.

CPES. 2007. Collaborative Psychiatric Epidemiology Surveys (CPES), 2001-2003 User's Guide. Available online: http:/ / www.icpsr.umich.edu/icpsrweb/ICPSR/studies/20240 (accessed on 31 March 2017).

CPES. 2008. CPES Weights Charts. Available online: http://www.icpsr.umich.edu/files/CPES/CPES\% 20Weights\%20Chart.pdf (accessed on 31 March 2017).

Das, Ajit K., and Sharon F. Kemp. 1997. Between Two Worlds: Counseling South Asian Americans. Journal of Multicultural Counseling and Development 25: 23-33. [CrossRef]

Fong, Timothy P. 2007. The Contemporary Asian American Experience: Beyond the Model Minority, 3rd ed. Upper Saddle River: Pearson.

Gao, Ling-ling, Sally Wai-chi Chan, and Qing Mao. 2009. Depression, Perceived Stress, and Social Support among First-Time Chinese Mothers and Fathers in the Postpartum Period. Research in Nursing $\mathcal{E}$ Health 32: 50-58.

Gee, Gilbert C., Michael Spencer, Juan Chen, Tiffany Yip, and David T. Takeuchi. 2007. The Association between Self-Reported Racial Discrimination and 12-Month DSM-IV Mental Disorders among Asian Americans Nationwide. Social Science \& Medicine 64: 1984-96.

Goebert, Deborah. 2009. Social Support, Mental Health, Minorities, and Acculturative Stress. In Determinants of Minority Mental Health and Wellness. Edited by S. Loue and M. Sajatovic. New York: Springer, pp. 1-24.

Greenberger, Ellen, and Chuansheng Chen. 1996. Perceived Family Relationships and Depressed Mood in Early and Late Adolescence: A Comparison of European and Asian Americans. Developmental Psychology 32: 707-16. [CrossRef]

Heeringa, Steven G., James Wagner, Myriam Torres, Naihua Duan, Terry Adams, and Patricia Berglund. 2004. Sample Designs and Sampling Methods for the Collaborative Psychiatric Epidemiology Studies (CPES). International Journal of Methods in Psychiatric Research 13: 221-40. [CrossRef] [PubMed]

Hoeffel, Elizabth M., Sonya Rastogi, Myoung Ouk Kim, and Hasan Shahid. 2012. The Asian Population: 2010. In 2010 Census Briefs; Suitland: U.S. Census Bureau.

Huang, Frederick Y., Henry Chung, Kurt Kroenke, Kevin L. Delucchi, and Robert L. Spitzer. 2006. "Using the Patient Health Questionnaire-9 to Measure Depression among Racially and Ethnically Diverse Primary Care Patients". Journal of General Internal Medicine 21(6): 547-52. [CrossRef] [PubMed]

Hwang, Wei-Chin, Hector F. Myers, and David T. Takeuchi. 2000. Psychosocial Predictors of First-Onset Depression in Chinese Americans. Social Psychiatry and Psychiatric Epidemiology 35(3): 133-45. [CrossRef]

Hwu, Hao-Gwo, I-Hao Chang, Eng-Kung Yeh, Ching-Jui Chang, and Ling-Ling Yeh. 1996. Major Depressive Disorder in Taiwan Defined by the Chinese Diagnostic Interview Schedule. The Journal of Nervous and Mental Disease 184(8): 497-502. [CrossRef]

Jackson, James S., Myriam Torres, Cleopatra H. Caldwell, Harold W. Neighbors, Randolph M. Nesse, Robert Joseph Taylor, Steven J. Trierweiler, and David R. Williams. 2004. The National Survey of American Life: A Study of Racial, Ethnic and Cultural Influences on Mental Disorders and Mental Health. International Journal of Methods in Psychiatric Research 13: 196-207. [CrossRef] [PubMed]

Juang, Linda P., and Alvin A. Alvarez. 2010. Discrimination and Adjustment among Chinese American Adolescents: Family Conflict and Family Cohesion as Vulnerability and Protective Factors. American Journal of Public Health 100: 2403-9. [CrossRef] [PubMed] 
Juang, Linda P., Moin Syed, and Miyuki Takagi. 2007. Intergenerational Discrepancies of Parental Control among Chinese American Families: Links to Family Conflict and Adolescent Depressive Symptoms. Journal of Adolescence 30: 965-75. [CrossRef] [PubMed]

Kalibatseva, Zornitsa, and Frederick T. Leong. 2011. Depression among Asian Americans: Review and Recommendations. Depression Research and Treatment 2011: 9. [CrossRef] [PubMed]

Kessler, Ronald C., and T. Bedirhan Üstün. 2004. The World Mental Health (WMH) Survey Initiative version of the World Health Organization (WHO) Composite International Diagnostic Interview (CIDI). International Journal of Methods in Psychiatric Research 13: 93-121. [CrossRef] [PubMed]

Kessler, Ronald C., Katherine A. McGonagle, Shanyang Zhao, Christopher B. Nelson, Michael Hughes, Suzann Eshleman, Hans-Ulrich Wittchen, and Kenneth Kendler. 1994. Lifetime and 12-Month Prevalence of DSM-III-R Psychiatric Disorders in the United States Results from the National Comorbidity Survey. Archives of General Psychiatry 51: 8-19. [CrossRef] [PubMed]

Kessler, Ronald C., Patricia Berglund, Wai Tat Chiu, Olga Demler, Steven Heeringa, Eva Hiripi, Robert Jin, Beth-Ellen Pennell, Ellen E. Walters, Alan Zaslavsky, and Hui Zheng. 2004. The US National Comorbidity Survey Replication (NCS-R): Design and Field Procedures. International Journal of Methods in Psychiatric Research 13: 69-92. [CrossRef]

Kim, Hyoun K., and Patrick C. McKenry. 2002. The Relationship between Marriage and Psychological Well-being A Longitudinal Analysis. Journal of Family Issues 23: 885-911. [CrossRef]

Kim, Bryan S. K., Bradley R. Brenner, Christopher T. H. Liang, and Penelope A. Asay. 2003. A Qualitative Study of Adaptation Experiences of 1.5-Generation Asian Americans. Cultural Diversity E Ethnic Minority Psychology 9: 156-70.

Kleinman, Arthur M. 1977. Depression, Somatization and the "New Cross-cultural Psychiatry". Social Science $\mathcal{E}$ Medicine 11: 3-9.

Kleinman, Arthur M. 1982. Neurasthenia and Depression: A Study of Somatization and Culture in China. Culture, Medicine and Psychiatry 6: 117-90. [CrossRef] [PubMed]

Kriegsman, Didi M. W., Brenda W. J. H. Penninx, Jacques Th. M. Van Eijk, A. Joan P. Boeke, and Dorly J. H. Deeg. 1996. Self-Reports and General Practitioner Information on the Presence of Chronic Diseases in Community Dwelling Elderly. Journal of Clinical Epidemiology 49: 1407-17. [CrossRef]

Kuo, Wen H. 1984. Prevalence of Depression among Asian-Americans. The Journal of Nervous and Mental Disease 172: 449-57. [CrossRef] [PubMed]

Lai, Daniel W. 2004. Depression among Elderly Chinese-Canadian Immigrants from Mainland China. Chinese Medical Journal 117: 677-83. [PubMed]

Lee, Minsun. 2011. Generational Diversity among Immigrants. The Clinical Psychologist 64: 11-12.

Lee, Sing, S. C. Fung, A. Tsang, Z. R. Liu, Y. Q. Huang, Y. L. He, M. Y. Zhang, Y. C. Shen, M. K. Nock, and Ronald C. Kessler. 2007. Lifetime Prevalence of Suicide Ideation, Plan, and Attempt in Metropolitan China. Acta Psychiatrica Scandinavic 116: 429-37. [CrossRef] [PubMed]

Lee, Sing, A. Tsang, Y. Q. Huang, Y.-L. He, Z. R. Liu, M.-Y. Zhang, Y.-C. Shen, and Ronald C. Kessler. 2009. The Epidemiology of Depression in Metropolitan China. Psychological Medicine 39: 735-47. [CrossRef] [PubMed]

Lee, Minsun, David T. Takeuchi, Zvi Gellis, Philip Kendall, Lin Zhu, Shanyang Zhao, and Grace X. Ma. 2016. The Impact of Perceived Need and Relational Factors on Mental Health Service Use among Generations of Asian Americans. Journal of Community Health, 1-13. [CrossRef] [PubMed]

Leu, Janxin, Irene H. Yen, Stuart A. Gansky, Emily Walton, Nancy E. Adler, and David T. Takeuchi. 2008. The Association between Subjective Social Status and Mental Health among Asian Immigrants: Investigating the Influence of Age at Immigration. Social Science \& Medicine 66: 1152-64.

Leung, C. M., K. K. Chan, and K. K. Cheng. 1992. Psychiatric morbidity in a general medical ward. Hong Kong's experience. General Hospital Psychiatry 14: 196-200. [CrossRef]

Lieber, Eli, Dorothy Chin, Kazuo Nihira, and Iris T. Mink. 2001. Holding on and Letting Go: Identity and Acculturation among Chinese Immigrants. Cultural Diversity and Ethnic Minority Psychology 7: 247-61. [CrossRef] [PubMed]

Lin, Nan. 1989. Measuring Depressive Symptomatology in China. Journal of Nervous and Mental Disease 177: 121-31. [CrossRef] [PubMed]

Marsella, Anthony J. 1978. Thoughts on Cross-Cultural Studies on the Epidemiology of Depression. Culture, Medicine and Psychiatry 2: 343-57. [PubMed] 
Mezzich, Juan E., Laurence J. Kirmayer, Arthur M. Kleinman, Horatio Fabrega, Delores L. Parron, Buron J. Good, Keh-Ming Lin, and Spero M. Manson. 1999. The Place of Culture in DSM-IV. The Journal of Nervous and Mental Disease 187: 457-64. [CrossRef] [PubMed]

Mui, Ada C. 1999. Living Alone and Depression among Older Chinese Immigrants. Journal of Gerontological Social Work 30: 147-66. [CrossRef]

Mulvaney-Day, Norah E., Margarita Alegría, and William Sribney. 2007. Social Cohesion, Social Support, and Health among Latinos in the United States. Social Science E Medicin 64: 477-95.

Nguyen, Duy, and Lindsay A. Bornheimer. 2014. Mental Health Service Use Types among Asian Americans with a Psychiatric Disorder: Considerations of Culture and Need. The Journal of Behavioral Health Services E Research 41: 520-28.

Nicdao, Ethel G., Seunghye Hong, and David T. Takeuchi. 2008. Social Support and the Use of Mental Health Services among Asian Americans: Results from the National Latino and Asian American Study. Research in the Sociology of Health Care 26: 167-84.

Obradović, Jelena, Nicole Tirado-Strayer, and Janxin Leu. 2013. The Importance of Family and Friend Relationships for the Mental Health of Asian Immigrant Young Adults and Their Nonimmigrant Peers. Research in Human Development 10: 163-83.

Park, Kyeyoung. 1999. "I Really Do Feel I'm 1.5!": The Construction of Self and Community by Young Korean Americans. Amerasia Journal 25: 139-63. [CrossRef]

Portes, Alejandro, and Rubén G. Rumbaut. 2001. Legacies: The Story of the Immigrant Second Generation. Oakland: University of California Press.

Qin, Desirée Boalian, Niobe Way, and Preetika Mukherjee. 2008. The Other Side of the Model Minority Story the Familial and Peer Challenges Faced by Chinese American Adolescents. Youth E Society 39: 480-506.

Radloff, Lenore S. 1977. The CES-D Scale A Self-Report Depression Scale for Research in the General Population. Applied Psychological Measurement 1: 385-401. [CrossRef]

Rumbaut, Rubén G. 1994. The Crucible within: Ethnic Identity, Self-Esteem, and Segmented Assimilation among Children of Immigrants. The International Migration Review 28: 748-94. [CrossRef]

Salant, Talya, and Diane S. Lauderdale. 2003. Measuring Culture: A Critical Review of Acculturation and Health in Asian Immigrant Populations. Social Science \& Medicine 57: 71-90.

Sangalang, Cindy C., and Gilbert C. Gee. 2012. Depression and Anxiety among Asian Americans: The Effects of Social Support and Strain. Social Work 57: 49-60. [CrossRef] [PubMed]

Schmid, Carol L. 2001. Educational Achievement, Language-Minority Students, and the New Second Generation. Sociology of Education 74: 71-87. [CrossRef]

Shen, Biing-Jiun, and David T. Takeuchi. 2001. A Structural Model of Acculturation and Mental Health Status among Chinese Americans. American Journal of Community Psychology 29: 387-418. [CrossRef] [PubMed]

StataCorp. 2015. Stata Statistical Software: Release 14. College Station: StataCorp LP.

Stice, Eric, Jennifer Ragan, and Patrick Randall. 2004. Prospective Relations between Social Support and Depression: Differential Direction of Effects for Parent and Peer Support? Journal of Abnormal Psychology 113: 155-59. [CrossRef] [PubMed]

Tabora, Betty, and Jacquelyn H. Flaskerud. 1994. Depression among Chinese Americans: A Review of the Literature. Issues in Mental Health Nursing 15: 569-84. [CrossRef] [PubMed]

Takeuchi, David T., Rita Chi-Ying Chung, Keh-Ming Lin, Haikang Shen, Karen Kurasaki, Chi-Ah Chun, and Stanley Sue. 1998. Lifetime and Twelve-Month Prevalence Rates of Major Depressive Episodes and Dysthymia among Chinese Americans in Los Angeles. American Journal of Psychiatry 155: 1407-14. [CrossRef] [PubMed]

Takeuchi, David T., Nolan Zane, Seunghye Hong, David H. Chae, Fang Gong, Gilbert C. Gee, Emily Walton, Stanley Sue, and Margarita Alegría. 2007. Immigration-Related Factors and Mental Disorders among Asian Americans. American Journal of Public Health 97: 84-90. [CrossRef] [PubMed]

Tsai, Jeanne L., and Robert W. Levenson. 1997. Cultural Influences on Emotional Responding Chinese American and European American Dating Couples during Interpersonal Conflict. Journal of Cross-Cultural Psychology 28: 600-25. [CrossRef]

Tseng, Wen-Shing, and Jing Hsu. 1970. Chinese Culture, Personality Formation and Mental Illness. International Journal of Social Psychiatry 16: 5-14. [CrossRef]

Uba, Laura. 2003. Asian Americans: Personality Patterns, Identity, and Mental Health. New York: Guilford Press. 
Waters, Mary C., and Tomás R. Jiménez. 2005. Assessing Immigrant Assimilation: New Empirical and Theoretical Challenges. Annual Review of Sociology 31: 105-25. [CrossRef]

Wei, Meifen, Tsun-Yao Ku, Daniel W. Russell, Brent Mallinckrodt, and Kelly Yu-Hsin Liao. 2008. Moderating Effects of Three Coping Strategies and Self-Esteem on Perceived Discrimination and Depressive Symptoms: A Minority Stress Model for Asian International Students. Journal of Counseling Psychology 55: 451-62. [CrossRef] [PubMed]

Willgerodt, Mayumi Anne, and Elaine Adams Thompson. 2006. Ethnic and Generational Influences on Emotional Distress and Risk Behaviors among Chinese and Filipino American Adolescents. Research in Nursing $\mathcal{E}$ Health 29: 311-24.

Wu, Bei, Thanh V. Tran, and Quartel-Ayne Amjad. 2004. Chronic Illnesses and Depression among Chinese Immigrant Elders. Journal of Gerontological Social Work 43: 79-95. [CrossRef]

Ying, Yu-Wen. 1988. Depressive Symptomatology among Chinese-Americans as Measured by the CES-D. Journal of Clinical Psychology 44: 739-46. [CrossRef]

Ying, Yu-Wen, and Meekyung Han. 2007. The Longitudinal Effect of Intergenerational Gap in Acculturation on Conflict and Mental Health in Southeast Asian American Adolescents. American Journal of Orthopsychiatry 77: 61-66. [CrossRef] [PubMed]

Young, Christina B., Daniel Z. Fang, and Sidney Zisook. Depression in Asian-American and Caucasian Undergraduate Students. Journal of Affective Disorders 125: 379-82. [CrossRef] [PubMed]

Zhang, Amy Y., Lucy C. Yu, Jianping Yuan, Zhifu Tong, Chaoyuan Yang, and Stephen E. Foreman. 1997. Family and Cultural Correlates of Depression among Chinese Elderly. International Journal of Social Psychiatry 43: 199-212. [CrossRef] [PubMed]

Zhou, Min. 1997. Segmented Assimilation: Issues, Controversies, and Recent Research on the New Second Generation. The International Migration Reviews 31: 975-1008. [CrossRef]

(C) 2017 by the author. Licensee MDPI, Basel, Switzerland. This article is an open access article distributed under the terms and conditions of the Creative Commons Attribution (CC BY) license (http:/ / creativecommons.org/licenses/by/4.0/). 